\title{
Approaches for Mathematical Modeling and Experimental Study of the Turbulent Flows of Mutually Immiscible Liquids (Oil-Water)
}

\author{
Ivan V. Kazachkov \\ Dept of Information technology and data analysis, Nizhyn Gogol state university, Grafs'ka, 2, Ukraine, \\ Dept of Energy Technology, Royal Institute of Technology, Sweden
}

*Corresponding Author: Ivan V. Kazachkov, Dept of Information technology and data analysis, Nizhyn Gogol state university, Grafs'ka, 2, Ukraine, Dept of Energy Technology, Royal Institute of Technology, Sweden

\begin{abstract}
The oil-water flows are multiphase because these liquids are mutually immiscible; therefore such flows are difficult for mathematical modeling and experimentalstudy. Peculiarities of the turbulent two-phase and multiphase flows of the mutually immiscible liquids and averaged differential equations for their modeling are considered. Such equations were first derived by Prof. A.I. Nakorchevski as an alternative to a number of the well-known averaged multiphase dynamics equations. The main difference of the new method was in an averaging of the Navier-Stokes equations by phases and components in time instead of the widely spread spatial averaging in multiphase mechanics. What was more, the introduction of the so-called functionindicator of the phases in a flow allowed recognizing the phases in their movement in a multiphase mixture, both theoretically and experimentally. For the experimentalstudy, the special micro sensor was invented and created, which was successfully applied. In this paper, the method of Nakorchevski is described in application to some multiphase tasks, and a discussion is presented as concern to advantages of the method for study of turbulent two-phase flows of water-oil immiscible mixture, as well as many other similar mixtures, where it is important to know the peculiarities of the phases' movement and their mixing in a multiphase flow.
\end{abstract}

Keywords: Mutually Immiscible Liquids; Mixing; Trajectories of Phases; Modeling; Time Averaging

\section{INTRODUCTION AND METHODOLOGY}

\subsection{The Method of Prof. A.I. Nakorchevski for Heterogeneous Turbulent Jets}

The first attempt to develop a method, which naturally reflects the most important features of the flows of two or more immiscible liquids like a water and an oil was the one by Prof. A.I. Nakorchevski [1]. The characteristics of the mixture $a^{l}(t)$ (mass, velocity, impulse, etc.) of the corresponding characteristics of different phases $a_{i}^{l}(t)$ in a multiphase flow was proposed to be expressed as follows

$a^{l}(t)=\sum_{i=1}^{m} B_{i}(t) a_{i}^{l}(t)$

where $B_{i}(t)$ was introduced as co-called function-indicator determined as

$B_{i}(t)=\left\{\begin{array}{l}1, \text { if } i \text {-phase occupies the elementary volume } \delta \mathrm{V} \\ 0, \text { if } i \text {-phase is outside the elementary volume } \delta \mathrm{V}\end{array}\right.$.

With this approach, the analog of the Navier-Stokes equations in a boundary layer approximation was derived:

$$
\begin{aligned}
& \frac{\partial}{\partial x}\left(y \rho_{i} B_{i} u_{i}\right)+\frac{\partial}{\partial y}\left(y \rho_{i} B_{i} v_{i}\right)=0, \quad \sum_{i=1}^{m} B_{i}=1, \\
& \rho_{i} B_{i}\left(u_{i} \frac{\partial u_{i}}{\partial x}+v_{i} \frac{\partial u_{i}}{\partial y}\right)=-\frac{d p}{d x}+\frac{1}{y} \frac{\partial}{\partial y}\left[y B_{i} \tau_{i}\right]_{m},
\end{aligned}
$$


Approaches for Mathematical Modeling and Experimental Study of the Turbulent Flows of Mutually Immiscible Liquids (Oil-Water)

where is the sum by mute index $i$, which belong to a phase $i$. In the stationary equations of incompressible liquids (3) written in a cylindrical coordinate system are: $p$ - pressure, $\rho$ - density, $u, v$ the longitudinal and transversal velocity components, $\tau_{i}$ - turbulent stress for a phase $i$. Index $m$ belongs to the values at the axis of the flow (symmetry axis). All values are averaged on the given interval by time.

\subsection{Schematic Representation of Turbulent Jet of Two Immiscible Liquids}

The schematic representation of the turbulent two-phase flow of two immiscible liquids is given in Fig. 1:

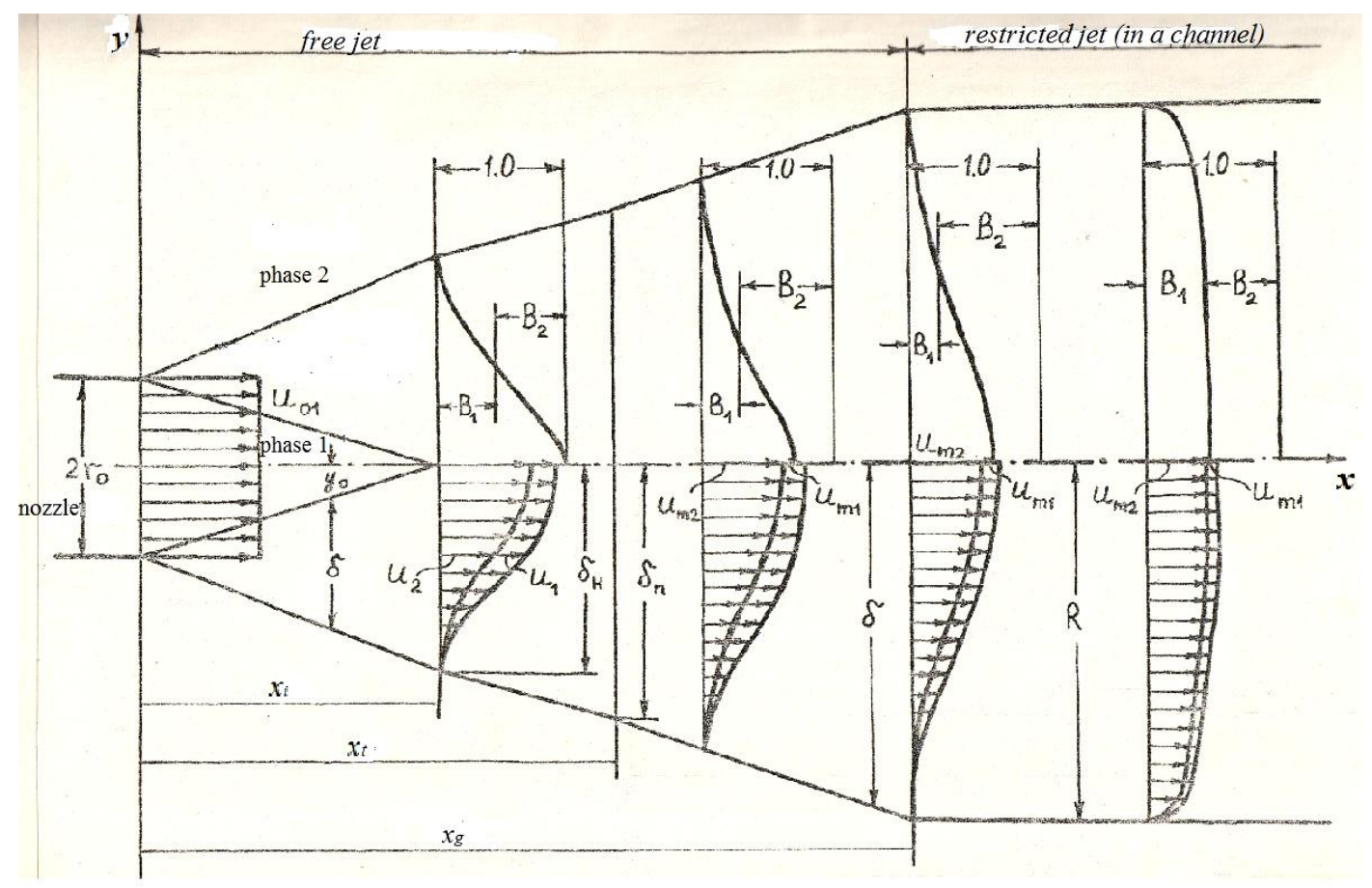

Fig1. The structural scheme of the two phase jet

One liquid flow is going from the nozzle of the radius $r_{0}$ with velocity $u_{01}$ (the velocity profile is supposed simple uniform) in surroundings occupied by other liquid (phase 2) being in a rest. The structure of a jets mixing with surrounding liquid according to the Fig. 1 is simplified according to a traditional scheme [2]. First, the initial part of the length $x_{\mathrm{i}}$ with the approximately linear boundaries for the conical surface (in cylindrical coordinates) of the internal potential core of a first phase and the external interface (conical too) are considered as the boundaries of the mixing area and the central potential core, correspondingly. The mixing turbulent zone between the above surfaces contains drops and fragments of the phases as far as immiscible liquids have behaviors like the separate phases, with their interfacial multiple surfaces interacting in all such locations (exchange of mass, impulse, energy among the phases).Close to the external interface, there is a flow of a surrounding liquid with some drops of the first phase, while, in turn, close to the boundary of the potential core there is a flow of the first phase with the drops of a second phase. As far as we have mutually immiscible liquids, the mixing zone contains two phase mixture in a turbulent flow. After an initial part of the mixing zone when all first phase in a potential core is spent, the short transit area is preparing the ground part of the turbulent two-phase jet, where both phases are well mixed across all layer.

Normally for description of multiphase flows the spatial averaging of the differential equations of mass, impulse and energy conservation is performed using the concept of volumetric phase content [3-6], which does not fit so well to experimental study of the separate phase movement in a mixture as the approach proposed in [1], where the special experimental technology and micro sensor for some measurements in two-phase flows has been developed as well. In [4, 5] fundamentals and analysis of the different methods for modeling of the multiphase systems can be found. Actually, all known methods of multiphase methods are well connected including the one in [1], and the parameters averaged by time can be easily transformed to the ones by [3-6]. 


\subsection{The Function-Indicator of the Phases and Parameters of Heterogeneous Flow}

The external interface of the mixing zone is determined zero longitudinal velocity of the second phase and transversal velocity of the first phase (the second phase is sucked from immovable surrounding into the mixing zone). The function-indicator of the first phase $B_{1}(t)$ is zero at the external interface because the first phase is absent in surroundings. Similar, the function-indicator $B_{2}(t)$ is zero on the interface of the potential core, the boundary of the first phase going from the nozzle. In a first approach, an influence of the mass, viscous and capillary forces are neglected. With an account of the above-mentioned, the boundary conditions are stated as follows [1]:

$y=y_{0}, u_{i}=u_{0 i}, v_{i}=0, \tau_{i}=0, B_{1}=1 ; y=y_{0}+\delta, u_{i}=0, v_{i}=0, \tau_{i}=0, B_{1}=0$.

The turbulent stress in the phase is stated by the "new" Prandtl's formula

$\tau_{i}=\rho_{i} \kappa_{i} \delta u_{m i} \partial u_{i} / \partial y$

where $\kappa_{i}$ is the coefficient of turbulent mixing for $i$-th phase, $\delta$ is the width of the mixing layer.

\subsection{The Polynomial Approximations of the Flow Parameters Across the Layer of a Jet}

The polynomial approximations for the velocity profiles and other functions in the turbulent mixing zone have been obtained based on the boundary conditions (4) in the form:

$$
\begin{aligned}
& u_{1} / u_{01}=1-4 \eta^{3}+3 \eta^{4}, \\
& u_{2} / u_{02}=1-6 \eta^{2}+8 \eta^{3}-3 \eta^{4}, \\
& B_{1}=B_{1}^{(0)}=1-\eta^{3}+0.5 \eta^{2}(1-\eta) h(x), \quad h \in[-6,0], \\
& B_{1}=B_{1}^{(1)}=1-4 \eta^{3}+3 \eta^{4}+0.5 \eta^{2}(1-\eta)^{2} h(x), \quad h \in[-12,-6], \\
& B_{1}=B_{1}^{(2)}=1-10 \eta^{3}+15 \eta^{4}-6 \eta^{5}+0.5 \eta^{2}(1-\eta)^{3} h(x), \quad h \in[-20,-12], \\
& B_{1}=B_{1}^{(3)}=1-20 \eta^{3}+45 \eta^{4}-36 \eta^{5}+10 \eta^{6}+0.5 \eta^{2}(1-\eta)^{4} h(x), \quad h \in[-30,-20], \\
& B_{1}=B_{1}^{(4)}=1-35 \eta^{3}+105 \eta^{4}-126 \eta^{5}+70 \eta^{6}-15 \eta^{7}+0.5 \eta^{2}(1-\eta)^{5} h(x), \quad h \in[-42,-30] \\
& B_{1}^{(5)}=1-56 \eta^{3}+210 \eta^{4}-336 \eta^{5}+280 \eta^{6}-120 \eta^{7}+21 \eta^{8}+0.5 \eta^{2}(1-\eta)^{6} h(x), \quad h \in[-56,-42] \\
& B_{1}^{(6)}=1-84 \eta^{3}+378 \eta^{4}-756 \eta^{5}+840 \eta^{6}-540 \eta^{7}+189 \eta^{8}-29 \eta^{9}+0.5 \eta^{2}(1-\eta)^{7} h(x), \quad h \in[-72,-56],
\end{aligned}
$$

where $h(x)=\left(\partial^{2} B_{1} / \partial \eta^{2}\right)_{\eta=0}$ is an interesting function, which determines a transition of the piecewise continuous function-indicator $B_{1}^{(n)}$ to its next approximation, determined from the condition that the derivative by with respect to a point $\eta=1$ be equal to zero up to $(n+1)$-th and including order.

\section{Governing Equations for the Heterogeneous Two-PhaSe Jet}

\subsection{Integral Correlations for the Initial Part of a Jet Flow}

Based on the above considered approximations the integral correlations have been derived for the two-phase turbulent jet on the initial part according to the structural scheme in Fig. 1 [1]:

$$
\begin{aligned}
& u_{01}\left(r_{0}^{2}-y_{0}^{2}\right)=2 \delta \int_{0}^{1} B_{1} u_{1}\left(y_{0}+\delta \eta\right) d \eta, \\
& \rho_{1} u_{01}^{2}\left(r_{0}^{2}-y_{0}^{2}\right)=2 \delta \int_{0}^{1}\left(\rho_{1} B_{1} u_{1}^{2}+\rho_{2} B_{2} u_{2}^{2}\right)\left(y_{0}+\delta \eta\right) d \eta, \\
& \rho_{1} u_{01}\left(u_{01}-u_{1}^{*}\right) y_{0} y_{0}^{\prime}+\frac{d}{d x} \delta \int_{0}^{\eta^{*}} \sum_{j=1}^{2} \rho_{j} B_{j} u_{j}^{2}\left(y_{0}+\delta \eta\right) d \eta-\sum_{j=1}^{2} u_{i}^{*} \frac{d}{d x} \delta \int_{0}^{\eta^{*}} \rho_{i} B_{i} u_{i}\left(y_{0}+\delta \eta\right) d \eta=
\end{aligned}
$$




$$
=\left(y_{0}+\delta \eta^{*}\right) \sum_{j=1}^{2} \rho_{j} B_{j} \kappa_{j} u_{0 j} \frac{\partial u_{j}^{*}}{\partial \eta}, \quad B_{1}+B_{2}=1 .
$$

The first equation in (9) was got integrating by $y$ the mass conservation equation, the second and the third ones - integrating the impulse conservation for the total flow of a two-phase mixture for $y=y_{0}+\delta$ and $y=y^{*}$, respectively. The polynomial approximations for the functions $u_{2}, B_{1}$ on a ground part of the jet keep the same but for the function $u_{l}$ approximation is as follows

$$
u_{1} / u_{m 1}=1-3 \eta^{2}+2 \eta^{3}
$$

\subsection{Integral Correlations for the Ground Part of a Jet Flow}

The integral correlations for the ground part of a jet obtained similarly to the above described initial part are as follows [1]:

$$
\begin{aligned}
& 2 \int_{0}^{\delta} B_{1} u_{1} y d y=u_{01} r_{0}^{2}, 2 \sum_{j=1}^{2} \int_{0}^{\delta} \rho_{j} B_{j} u_{j}^{2} y d y=\rho_{1} u_{01}^{2} r_{0}^{2}, \\
& \frac{d}{d x} \sum_{j=1}^{2} \int_{0}^{y^{*}} \rho_{j} B_{j} u_{j}^{2} y d y-\sum_{j=1}^{2} u_{j}^{*} \frac{d}{d x} \int_{0}^{y^{*}} \rho_{j} B_{j} u_{j} y d y=y^{*} \sum_{j=1}^{2} B_{j}^{*} \tau_{j}^{*},
\end{aligned}
$$

where the first is the equation of the mass conservation for the first phase, the second and the third the momentum conservation equations for the total and for the part of the cross section, respectively, according to the methodology [2]. And the momentum equation on the jet's axis $(y=0)$ is used too:

$$
\sum_{j=1}^{2} \rho_{j} B_{m j} u_{m j} \frac{d u_{m j}}{d x}=2 \sum_{j=1}^{2}\left[\frac{\partial}{\partial y}\left(B_{j} \tau_{j}\right)\right]_{m} .
$$

The mathematical model including the ordinary differential equations (9), (11), (12) by longitudinal coordinate $x$ is implemented for analysis and numerical simulation on the computer the basic features of the stationary turbulent two-phase jet of two immiscible liquids. The function-indicator $B_{1}$ shows how much is a presence of the first phase in a selected point of mixing zone, which can be directly compared to an experimental data by the two-phase sensor. Therefore, a solution of the task may give both parameters of the flow together with their belonging to a particular phase.

\subsection{Dimensionless form of the outgoing equation array and the profiles of basic parameters}

The equation array (9) with the boundary conditions (4) is used for numerical simulation of the turbulent two-phase jet on its initial part. For this, the equations (9) are transformed to the following dimensionless form with the scales $r_{0}, \delta, u_{0 i}$ for the longitudinal and transversal coordinates and velocity, respectively:

$$
\begin{aligned}
& y_{0}^{2}+2 \delta \sum_{j=1}^{2} y_{0}^{2-j} \delta^{j-1} a_{j}=1, \quad y_{0}^{2}+2 \delta \sum_{j=1}^{2} y_{0}^{2-j} \delta^{j-1}\left(a_{j+2}+i_{0} b_{j+2}\right)=1 \\
& \left(1-u_{1}^{*}\right) y_{0} \frac{d y_{0}}{d \varsigma}+\frac{d}{d \varsigma} \delta \sum_{j=1}^{2} y_{0}^{2-j} \delta^{j-1}\left(a_{j+2}^{*}+i_{0} b_{j+2}^{*}\right)-\frac{d}{d \varsigma} \delta \sum_{j=1}^{2} y_{0}^{2-j} \delta^{j-1}\left(a_{j}^{*} u_{1}^{*}+i_{0} b_{j}^{*} u_{2}^{*}\right)=\left(y_{0}+\delta \eta^{*}\right)= \\
& =\left(y_{0}+\delta \eta^{*}\right) \sum_{j=1}^{2} B_{j}^{*}\left(\frac{\partial u_{j}}{\partial \eta}\right)^{*}\left(i_{0} \kappa_{21}\right)^{j-1}, \quad B_{1}+B_{2}=1 .
\end{aligned}
$$

Here are:

$$
\begin{aligned}
& \bar{y}_{0}=y_{0} / r_{0}, \bar{\delta}=\delta / r_{0}, \eta=\left(y-y_{0}\right) / \delta, \bar{x}=x / r_{0}, \varsigma=\kappa_{1} \bar{x}, s_{0}=u_{02} / u_{01}, i_{0}=n s_{0}^{2}, \\
& n=\rho_{2} / \rho_{1}, \kappa_{21}=\kappa_{2} / \kappa_{1}, a_{i}=a_{i 1}+a_{i 2} h, b_{i}=b_{i 1}+b_{i 2} h, \\
& a_{i}=\int_{0}^{1} B_{1} \bar{u}_{1} \eta^{j-1} d \eta, b_{i}=\int_{0}^{1} B_{2} \bar{u}_{2} \eta^{j-1} d \eta(i=1,2) ; a_{i}=\int_{0}^{1} B_{1} \bar{u}_{1}^{2} \eta^{j-1} d \eta, b_{i}=\int_{0}^{1} B_{2} \bar{u}_{2}^{2} \eta^{j-1} d \eta(i=3,4) ; j=1,2 .
\end{aligned}
$$


Except the above, for the dimensionless parameters, we retain the same notations as for the dimensional ones. Only here in (14) it is stated for clarification of the dimensionless notations.

The sliding factor $s_{0}$ is supposed to be constant and the values of the parameters at $\eta=\eta^{*}<1$ are signed with a star *. The system (13) must satisfy the following boundary conditions

$\zeta=0, \quad y_{0}=1, \delta=0 ; \zeta=\zeta_{i}, y_{0}=0, \quad \delta=\delta_{i}$;

where $\zeta_{i}, \delta_{i}$ are the dimensionless length of a jet and its maximal radius (at the end of the initial part).

By the computed from the above boundary problem (13), (15) functions $y_{0}(\zeta), \delta(\zeta), h(\zeta)$ we can find all the other characteristics of the jet flow for the stated values of the main parameters of the model $i_{0}$, $\kappa_{1}, \kappa_{2}$. The first parameter is slightly indefinite due to difficulties with exact estimation of the phases' sliding, while the other two are known from the experimental studies but only for specific conditions. In general, for each specific case the, the coefficients of turbulent mixing $\kappa_{1}, \kappa_{2}$ may be different, and it's the problem to estimate them correctly. This is the main problem with validation of the mathematical model using comparison with the experimental data. What is the main advantage here in the model, is the possibility to have all characteristics of a flow together with their belonging to a particular phase through the functions $B_{1}, B_{2}$.

The transversal velocities' distributions, interface interactions, the coefficients of the volumetric $q$ and mass ejection $g$ and kinetic energy $e_{i}$ for the phases in a flow are computed by the main characteristics obtained as above mentioned [1].For the short transient part of the jet there are no developed substantiated scheme, therefore it is not under consideration here, just short transient and then the ground part is considered, where the method is well elaborated and supported with the experimental data $[1,2]$. The dimensionless equation array for the ground part of the turbulent two-phase jet (11), (12) is the next

$$
\begin{aligned}
& 2 B_{m 1} u_{m 1} \delta^{2} \sum_{j=1}^{2} \alpha_{1 j} h^{j-1}=1,2 u_{m 1}^{2} \delta^{2}\left[B_{m 1} \sum_{j=1}^{2}\left(\alpha_{2 j}+i_{0} \beta_{2 j}\right) h^{j-1}+i_{0} \beta_{20}\right]=1 \\
& \frac{d}{d \varsigma} u_{m 1}^{2} \delta^{2}\left[B_{m 1} \sum_{j=1}^{2}\left(\alpha_{2 j}^{*}+i_{0} \beta_{2 j}^{*}\right) h^{j-1}+i_{0} \beta_{20}^{*}\right]-u_{m 1} \frac{d}{d \varsigma}\left(u_{m 1} \delta^{2}\right)\left[B_{m 1} \sum_{j=1}^{2}\left(\alpha_{1 j}^{*} u_{1}^{*}+i_{0} \beta_{1 j}^{*} u_{2}^{*}\right) h^{j-1}+i_{0} u_{2}^{*} \beta_{10}^{*}\right]= \\
& =\eta^{*} \delta u_{m 1}^{2}\left[\left(1-i_{0} \kappa_{21}\right) B_{m 1} \sum_{j=1}^{2}\left(u_{j}^{\prime}\right)^{*} \gamma_{j}^{*} h^{j-1}+i_{0} \kappa_{21}\left(u_{2}^{\prime}\right)^{*}\right]
\end{aligned}
$$

Here are:

$$
\begin{aligned}
& \bar{x}=\left(x-x_{t}\right) / r_{0}, i_{0}=n s_{0}^{2}, s_{0}=u_{m 2} / u_{m 1}, \bar{u}_{m i}=u_{m i} / u_{0 i}, \bar{u}_{i}=u_{i} / u_{m i}, \bar{B}_{2}=B_{2} / B_{m 1}, \\
& \bar{B}_{1}=B_{1} / B_{m 1}=\gamma_{1}+\gamma_{2} h, \int_{0}^{1} \bar{B}_{1} \bar{u}_{1}^{i} \eta d \eta=\sum_{j=1}^{2} \alpha_{i j} h^{j-1}, \int_{0}^{1} \bar{B}_{2} \bar{u}_{2}^{i} \eta d \eta=\beta_{i 0} / B_{m 1}+\beta_{i 1}+\beta_{i 2} h, \quad i=1,2 .
\end{aligned}
$$

A dash means a derivative by $\eta$, star * means a value by $\eta=\eta^{*}<1, x_{t}$ is the length of a transient part of a jet flow. It is assumed that $u_{\mathrm{m} 2}=s_{0} u_{\mathrm{m} 1}\left(s_{0}=\right.$ const), which means that sliding of the phases is preserved the same as for the initial part of a jet. For the dimensionless parameters, we retain the same notations as for the dimensional ones as before. The boundary condition for the equation array (17) are stated in a form

$\zeta=0, u_{m l}=1, B_{m l}=1, \delta=\delta_{t} ; \quad \zeta=¥, u_{m l}=0, B_{m l}=0, \quad \delta=¥ ;$

$\delta_{t}$ is a radius of the jet at the transient cross section.

\section{Solution of The Boundary Problem and Numerical Simulation}

\subsection{Basics of the Turbulent Heterogeneous Jet on Initial Part}

A solution of the boundary problem (16), (18) allows obtaining the functions $u_{m l}(\zeta), B_{m l}(\zeta), \delta(\zeta)$ and $h(\zeta)$ by the modeling parameters $i_{0}, \kappa_{21}$. And similar to the initial part, here it is also available to compute the distribution of all parameters of a flow. Computational experiments reveal basic features 
of the flows by the initial parameters and the conditions stated. For the initial part of a jet, the velocity distributions for the phases $\left(u_{1}, u_{2}\right)$ and function-indicators of the phases $\left(B_{1}, B_{2}\right)$ are stated, as well as the values of parameters at the cross section of the nozzle. The solution can be done as follows. The system (13) contains two algebraic and one differential equation. From the algebraic equation array the functions $y_{0}(h), \delta(h)$ are got, and then the differential equation is expressed in a standard form

$d h / d \zeta=\mathrm{F}\left(h(\zeta), i_{0}, \kappa_{21}\right)$,

prepared for a numerical solution. Certainly, the equation array (13) could be solved numerically in general but the way we applied is more comprehensive for understanding the basic features of the system, with as much as possible analytical expressions showing the explicit functions.

The range of the function's $h(\zeta)$ variation is determined by substitution of the boundary conditions in the functions $y_{0}(h), \delta(h)$, so that we obtain the next:

$$
\begin{aligned}
& y_{0}=\frac{1}{\sqrt{1+2 a_{1} \frac{a_{3}+i_{0} b_{3}-a_{1}}{a_{2}-i_{0} b_{4}-a_{4}}+2 a_{2}\left(\frac{a_{3}+i_{0} b_{3}-a_{1}}{a_{2}-i_{0} b_{4}-a_{4}}\right)^{2}}}, \delta=y_{0} \frac{a_{3}+i_{0} b_{3}-a_{1}}{a_{2}-i_{0} b_{4}-a_{4}} ; \\
& h_{0}=h(0)=\frac{a_{11}-a_{31}-i_{0} b_{31}}{a_{32}-a_{12}-i_{0} b_{32}}, \quad h_{i}=h\left(\varsigma_{i}\right)=\frac{a_{21}-a_{41}-i_{0} b_{41}}{a_{42}-a_{22}-i_{0} b_{42}} .
\end{aligned}
$$

$h_{0}\left(i_{0}\right), h_{i}\left(i_{0}\right)$ computed from the (21) are presented in Fig. 2. As it is observed, the functions have the breaks at the transition points of the permanent characteristic function $B_{1}^{(n)}(\eta, h)$ from the one regional approximation to the other one (a first derivative has break at those points). It is impossible to get a common approximation for the function $B_{l}(\eta, h)$ satisfying the boundary conditions in all range by parameter $i_{0}$ (due to requirement of variation of function $B_{l}$ in the range from 0 to 1 ). Calculations have shown that there no substantial difference in the final results of the numerical solution at the points of the function $B_{l}(\eta, h)$ transformation, so that this approach is attainable.

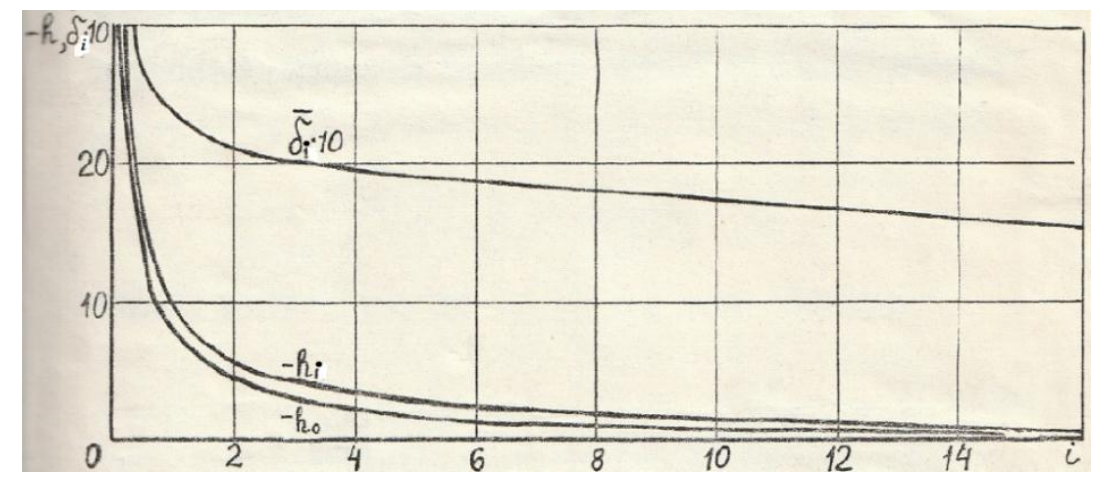

Fig2. Functions $h_{i}\left(i_{0}\right)$ and $\delta_{i}\left(i_{0}\right)$

Substitution of the values $h_{i}\left(i_{0}\right)$ from (21) into the equation (20) results in a relation for the radius of a jet at the end of the initial jet's part $\delta_{i}\left(i_{0}\right)$. The equations (20) determine the functions $y_{0}(h), \delta(h)$ in the range $h \in\left[h_{0}, h_{i}\right]$, which are monotonous and close to the linear ones by $i_{0}$ over 5 being rapidly falling by $i_{0}$ below 1 .

\subsection{Basics of the Turbulent Heterogeneous Jet on the Ground Part}

The ground part of a jet is described by the equations (16) with the boundary conditions (18). From the first two equations yields

$h=\frac{\alpha_{11}-\left(\alpha_{21}+i_{0} \beta_{21}\right) u_{m 1}-2 i_{0} \alpha_{11} \beta_{20} u_{m 1}^{2} \delta^{2}}{-\alpha_{12}+\left(\alpha_{22}+i_{0} \beta_{21}\right) u_{m 1}+2 i_{0} \alpha_{12} \beta_{20} u_{m 1}^{2} \delta^{2}}, B_{m 1}=\frac{2 i_{0}}{Z} \alpha_{12} \beta_{20}+\frac{\alpha_{22}+i_{0} \beta_{22}}{Z u_{m 1} \delta^{2}}-\frac{\alpha_{12}}{Z u_{m 1}^{2} \delta^{2}}$,

$Z=2\left[\left(\alpha_{11} \alpha_{22}-\alpha_{12} \alpha_{21}\right)+i_{0}\left(\alpha_{11} \beta_{22}-\alpha_{12} \beta_{21}\right)\right]$. The condition $\zeta=¥, B_{m l}=0$ leads the following: $\zeta=¥$, $u_{m l} \delta^{2}=¥, u_{m l} \delta=$ const, so that follows: 
$\lim _{\zeta \rightarrow \infty} \frac{\alpha_{12}}{Z}\left(2 i_{0} \beta_{20}-\frac{1}{u_{m 1}^{2} \delta^{2}}\right)=0$

where from with account of $\propto_{12} \neq 0$ yields

$\lim _{\zeta \rightarrow \infty} u_{m 1}^{2} \delta^{2}=\frac{1}{2 i_{0} \beta_{20}} \approx \begin{cases}33, & i_{0}=0.3 \\ 10, & i_{0}=1.0, h_{\infty}=\lim h \approx\left\{\begin{array}{ll}-70, & i_{0}=0.3 \\ 1.2, & i_{0}=8.0\end{array}{ }_{\zeta \rightarrow \infty}-i_{0}=1.0 .\right. \\ -18, & i_{0}=8.0\end{cases}$

The value $h_{\infty}$ is outside the region of the function's $h$ variation determined by $h=h_{t}$, where the ground part of a jet starts. Therefore function $B_{l}$ is continuous for each value $i_{0}$ (its first derivative is piecewise continuous). It is changing its approximation with transformation from the one to another region by $h$. Thus, after simple transformations the outgoing equation array yields the system of two ordinary first order differential equations with the corresponding boundary conditions:

$$
\begin{aligned}
& \frac{d u_{m 1}}{d \varsigma}=2 \frac{u_{m 1}}{\delta}\left(u_{1}^{\prime \prime}\right)_{0} \frac{\left(u_{1}^{\prime \prime}\right)_{0} B_{m 1}+i_{0} \kappa_{21}\left(1-B_{m 1}\right)\left(u_{2}^{\prime \prime}\right)_{0}}{\left(u_{1}^{\prime \prime}\right)_{0}\left[B_{m 1}+i_{0}\left(1-B_{m 1}\right)\right]}, \\
& \frac{d \delta}{d \varsigma}=\frac{1}{D_{1} u_{m 1}^{2} \delta}\left[\left(M_{1}+u_{m 1} \delta^{2} M_{2}+\frac{M_{3}}{u_{m 1}}\right) f_{1}+0.75\left(\frac{N_{3}}{\delta}+\frac{N_{2} u_{m 1}}{\delta}+N_{1} u_{m 1}^{2} \delta\right)\right] ; \\
& \varsigma=0, \quad u_{m 1}=1, \delta=\delta_{i} .
\end{aligned}
$$

Here are:

$$
\begin{aligned}
& M_{2}=\frac{2 i_{0}}{Z} \beta_{20}\left\{2\left[\alpha_{12}\left(\alpha_{21}^{*}+i_{0} \beta_{21}\right)-\left(\alpha_{22}^{*}+i_{0} \beta_{22}^{*}\right) \alpha_{11}\right]+\left[\alpha_{11}\left(\alpha_{12}^{*} u_{1}^{*}+i_{0} \beta_{12}^{*} u_{2}^{*}\right)-\alpha_{12}\left(\alpha_{11}^{*} u_{1}^{*}+i_{0} \beta_{12}^{*} u_{2}^{*}\right)\right]\right\}+ \\
& i_{0}\left(2 \beta_{20}^{*}-\beta_{10}^{*} u_{2}^{*}\right), \quad f=\frac{d u_{m 1}}{d \varsigma}, \quad M_{1}=\frac{1}{Z}\left[\left(\alpha_{21}^{*}+i_{0} \beta_{21}^{*}\right)\left(\alpha_{22}+i_{0} \beta_{22}\right)-\left(\alpha_{22}^{*}+i_{0} \beta_{22}^{*}\right)\left(\alpha_{21}+i_{0} \beta_{21}\right)\right], \\
& M_{3}=\frac{1}{Z}\left[\alpha_{11}\left(\alpha_{12}^{*} u_{1}^{*}+i_{0} \beta_{12}^{*} u_{2}^{*}\right)-\alpha_{12}\left(\alpha_{11}^{*} u_{1}^{*}+i_{0} \beta_{11}^{*} u_{2}^{*}\right)\right], \quad N_{3}=\frac{1}{Z}\left(\alpha_{12} \gamma_{1}-\alpha_{11} \gamma_{2}\right)\left(i_{0} \kappa_{21}-1\right), \\
& D_{1}=\frac{4 i_{0}}{Z} \beta_{20}\left[\alpha_{11}\left(\alpha_{22}^{*}+i_{0} \beta_{22}^{*}\right)-\alpha_{12}\left(\alpha_{21}^{*}+i_{0} \beta_{21}^{*}\right)-\alpha_{11}\left(\alpha_{12}^{*} u_{1}^{*}+i_{0} \beta_{12}^{*} u_{2}^{*}\right)+\alpha_{12}\left(\alpha_{11}^{*} u_{1}^{*}+i_{0} \beta_{11}^{*} u_{2}^{*}\right)\right]+ \\
& +2 i_{0}\left(\beta_{10}^{*} u_{2}^{*}-\beta_{20}^{*}\right), \quad N_{1}=\frac{2 i_{0}}{Z} \beta_{20}\left(\alpha_{12} \gamma_{1}-\alpha_{11} \gamma_{2}\right) i_{0} \kappa_{21}\left[1-\frac{2 i_{0}}{Z} \beta_{20}\left(\alpha_{12} \gamma_{1}-\alpha_{11} \gamma_{2}\right)\right], \\
& N_{2}=\frac{1}{Z}\left\{i_{0} \kappa_{21}\left[\gamma_{2}\left(\alpha_{12}+i_{0} \beta_{21}\right)-\gamma_{1}\left(\alpha_{22}+i_{0} \beta_{22}\right)\right]+\gamma_{1}\left(\alpha_{22}+i_{0} \beta_{22}\right)-\gamma_{2}\left(\alpha_{21}+i_{0} \beta_{21}\right)\right\} .
\end{aligned}
$$

\subsection{The Solution Procedure}

The boundary problem (23), (24) was solved numerically with a control of the value $h$ and automatic transforming of the approximation $B_{1}$. The functions $u_{m l}(\zeta), B_{m l}(\zeta), \delta(\zeta)=0$, and $h(\zeta)$ are computer for a range of parameters $i_{0}, \kappa_{21}$. The control parameters are kept in the computer program as follows: $B_{m l}<1, u_{m l}<1, \mathrm{~d} B_{m l} / \mathrm{d} \zeta<0, \mathrm{~d} \delta / \mathrm{d} \zeta>0$. An analysis of the numerical simulations has shown that by the boundary conditions (24) the solution is correct only for the restricted regions by a ratio of turbulent mixing coefficients $\kappa_{21}$ (specific for each value of the parameter $i_{0}$ ). It's an interesting feature that a ration of the turbulent mixing coefficients of the phase cannot be arbitrary, which seems to be physically reasonable. For the average value of the $\kappa_{21}^{a v}$ and the variation range $\Delta \kappa_{21}$ the following approximations were obtained:

$\kappa_{21}^{a v}=0.2 / i_{0}, \quad \Delta \kappa_{21}= \pm 0.02 i_{0}$.

The ratio of the coefficients of turbulent mixing is hyperbolically falling down with an increase of a density ratio: the higher is the density of an ejected liquid, the lower is its mixing coefficient 
comparing to a first liquid. But possible interval of a ratio can grow with the increase of the density ratio. It is understandable as heavy liquid loses its ability for intensive mixing with the other liquid.

An interesting feature was revealed about the influence of parameters $i_{0}, \kappa_{21}$ on the solution of the problem. The radius of a jet and velocities of phases on an axis practically don't depend on $\kappa_{21}$ being totally determined by the value $i_{0}$, while the functions $B_{m l}$ and $h$ strictly depend on $\kappa_{21}$. Thus, the turbulent mixing influences mostly the internal structure of a flow, phases' distribution, and velocities depend on density ratio of the phases (internal structure has little influence on it). The velocity distribution, in a turn, determines the radius of a mixing zone because it changes with falling of the velocity according to the mass and momentum conservation equations.

Due to the absence of the proven methodology for calculation of the transient part of a jet, the ground part of a turbulent two-phase jet is proposed for investigation in the following way, independent of limitations by $\kappa_{21}$. As far as two functions, $B_{m l}$ and $h$, determine the function $B_{l}$, and an influence of $B_{m l}$ is stronger than $h$, we can assume $h=h_{i}=$ const for the ground part of a jet so that the function $B_{m l}$ be correcting a possible inaccuracy of it. This assumption over determines the task, therefore we can have the possibility for calculation of the same characteristic twice, independently. We choose a radius of the mixing zone $\delta$. Controlling a ration of $\delta_{1}$ and $\delta_{2}$ obtained from the solution of two independent equations we can decide upon attainability of this our assumption, as well as total inaccuracy of the model. Then equations (22) yield:

$$
B_{m 1}=\beta_{20} \frac{u_{m 1} i_{0}}{\left(\alpha_{11}+\alpha_{12} h_{t}\right)-\left[\left(\alpha_{21}+i_{0} \beta_{21}\right)+\left(\alpha_{22}+i_{0} \beta_{22}\right) h_{t}\right] u_{m 1}}, \quad \delta=\frac{1}{\sqrt{2 B_{m 1} u_{m 1}\left(\alpha_{11}+\alpha_{12} h_{t}\right)}} .
$$

Then the equation array (23), (25) with the boundary conditions (24) was solved as follows. From the equations (25) and the first equation of the system (23), the functions $u_{m l}, B_{m l}$ and $\delta_{=} \delta_{1}$ are obtained depending on the longitudinal coordinate $\zeta$ and parameters $i_{0}, \kappa_{21}$. The other radius of the turbulent zone $\delta_{=} \delta_{2}$ is obtained from the second equation of the system (24) by $\eta=\eta^{*}=0.5$.

\subsection{The Results of Computer Simulations}

The results of computations for velocity, function-indicator of a phase and the radius of the turbulent mixing zone are given in the Fig. 3.The values $h_{i}\left(i_{0}\right), \delta_{i}\left(i_{0}\right)$ are presented in Fig. 4.

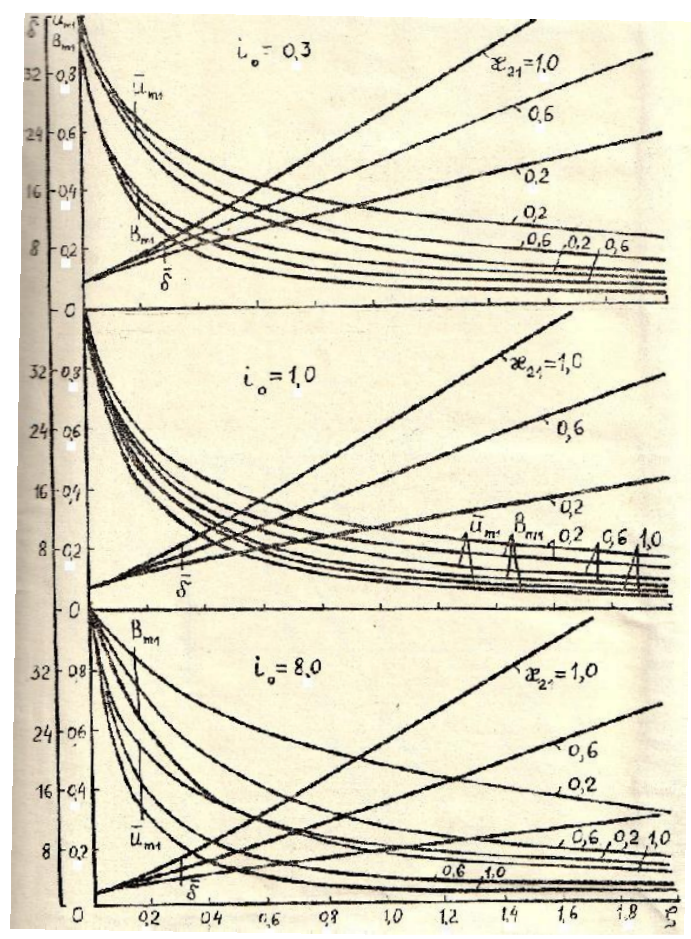

Fig3. Axial velocity, function-indicator and radius of the jet along the axis of a jet 
Approaches for Mathematical Modeling and Experimental Study of the Turbulent Flows of Mutually Immiscible Liquids (Oil-Water)

In the next calculations, the value $\delta_{=} \delta_{1}$ is accepted as a radius of a jet. Some of the computer simulations and the FORTRAN programs by the methodology described here first done by us were presented in [7-11]. Then it was further developed by Prof. Nakorchevki with his co-workers, as well as by other researchers in a number of papers, e.g. [12,13].

The equation (19) represented in a form

$\mathrm{d} h / \mathrm{d} \zeta=\mathrm{F}$,

After substitution of the computed functions $y_{0}(h) \delta(h)$, was solved in the following way. Function $\mathrm{FM}=1 / \mathrm{F}$ was computed in the range of parameters: $i_{0} \hat{\mathrm{I}}[0.2,16], \kappa_{21} \hat{\mathrm{I}}[0,5], h \hat{\mathrm{I}}[-20,0]$. Its approximation was found as $\mathrm{FM}=A_{1}{ }^{\prime}+A_{2}{ }^{\prime} h+A_{3}{ }^{\prime} h^{2}$, where $A_{\mathrm{i}}{ }^{\prime}\left(i_{0}, \kappa_{21}\right)$ - computed function with accuracy no less than $0.2 \%$. The maximal total inaccuracy of computation after integration of (26) estimated by the Caushy-Bunyakovski inequality satisfied the condition $\mathrm{D}_{\max }<\mathrm{D}_{\max }(\mathrm{D} h)_{\max }$. As far as the Fig. 2 shows $(\mathrm{D} h)_{\max }<4$, it was $\mathrm{D}_{\max }<0.8 \%$. It is within the attainable range of inaccuracy for the integral methods of the turbulent jets and boundary layers.

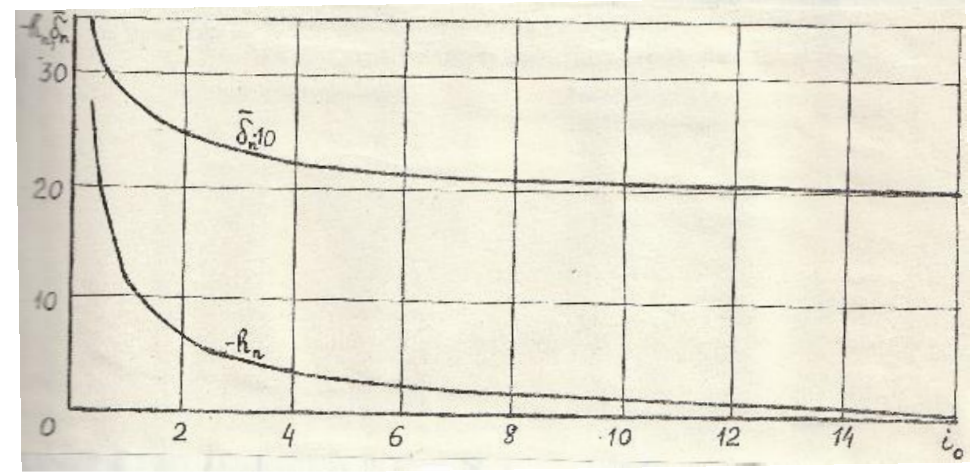

Fig4. Radius of a jet $\delta_{i}$ and function $h_{i}$ depending on parameter $i_{0}($ density ratio)

Integration of the equation (26) with account of the above-mentioned results

$\zeta=A_{0}+A_{1} h+A_{2} h^{2}+A_{3} h^{3}$,

where $A_{0}=-A_{1} h_{0}-A_{2} h_{0}^{2}-A_{3} h_{0}^{3}, A_{i}=A_{i}{ }^{\prime} / i, i=1,2,3$. Thus, $\zeta_{i}=A_{0}+A_{1} h_{i}+A_{2} h^{2}+A_{3} h^{3}{ }_{i}$. Computation by this formula is presented in Fig. 5:

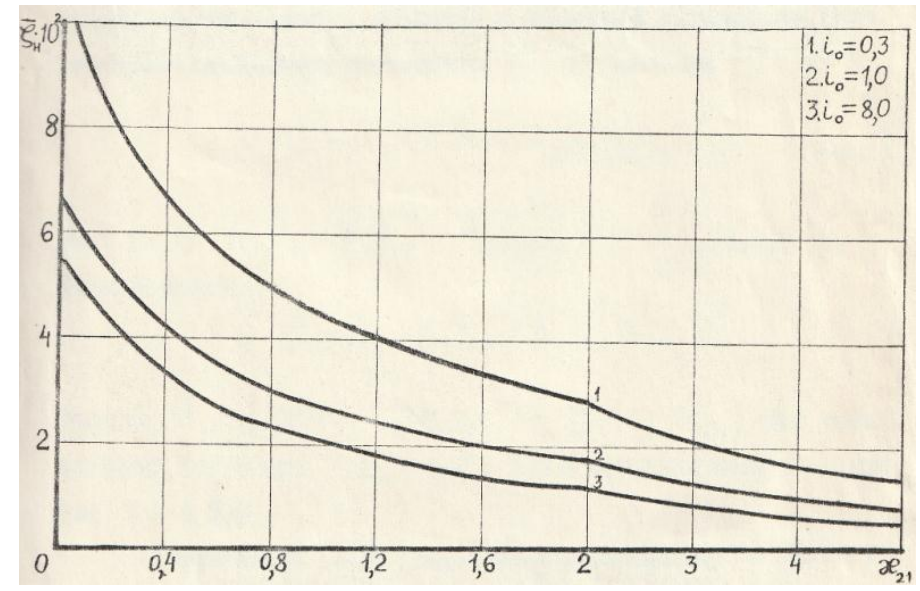

Fig5. The length of the initial part of a jet depending on the turbulent mixing ratio $\kappa_{21}$

where from follows that the length of the initial part of a jet strictly depends on a ratio of the turbulent mixing coefficients $\kappa_{21}$ : the higher is turbulent mixing in a second phase (ejected from the surrounding medium), the longer is an initial part of a jet. Strong mixing in a first phase coming from the nozzle means physically that it keeps parameters of the first phase for a long time. With a strong mixing coefficient of an injected phase, an initial part of a jet is short independent of density ration of the phases in a wide range from $i_{0}=0.3$ tilli $i_{0}=8$. If the mixing coefficients in phases are of the same 
Approaches for Mathematical Modeling and Experimental Study of the Turbulent Flows of Mutually Immiscible Liquids (Oil-Water)

order or coefficient in an ejected liquid is less than in a liquid going from the nozzle, the initial part of the jet is long (up to 5-10 times bigger than a radius of a nozzle). And the denser is first liquid, the long is an initial part of a jet.

The functions $A_{i}\left(i_{0}, \kappa_{21}\right), \zeta_{i}\left(i_{0}, \kappa_{21}\right), i=1,2,3$, for 3 different values of $i_{0}$ are presented in Figs 6, 7:

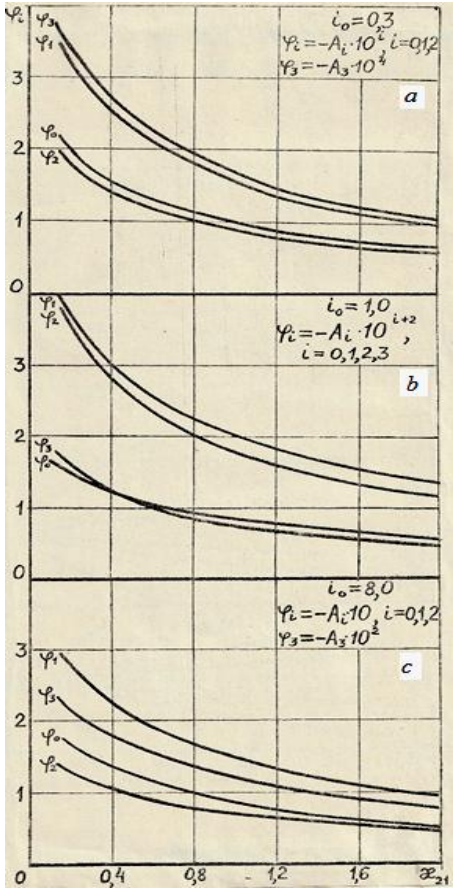

Fig6. The functions $A_{i}\left(i_{0}, \kappa_{21}\right)$ : dependence on $\kappa_{21}$ for 3 values of parameteri $i_{0}$

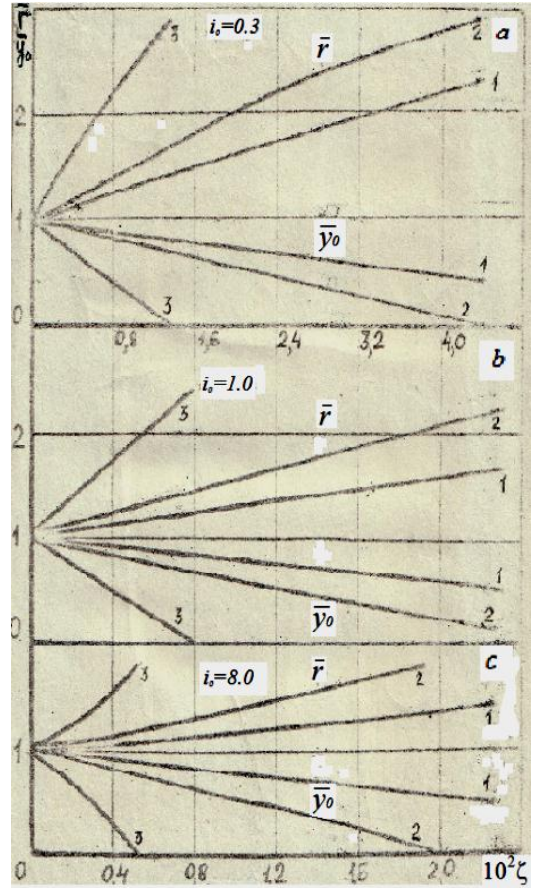

Fig7. Radius of potential core and mixing zone of twophase jet

The correlation (27) allows computing functions $y_{0}(h), \delta(h)$, and radius of a jet $r=y_{0}(h)+\delta(h)$, shown in Fig. $7\left(1-\kappa_{21}=0.2,2-\kappa_{21}=1,3-\kappa_{21}=5.0\right)$. The other characteristics of the turbulent jet are presented in Figs 8, 9:
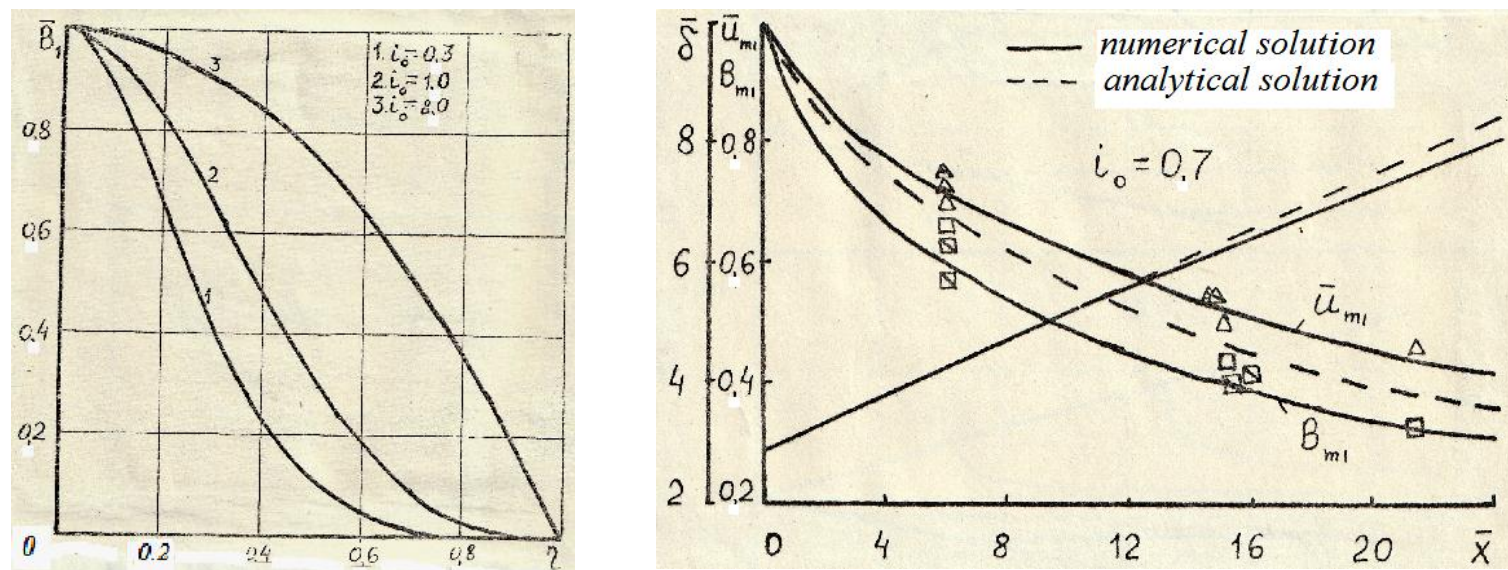

Fig8. The function-indicator $B_{1}$ and other parameters compared to experimental data

Comparison of the computer simulations done is shown in Fig. 8, where from can be observed that for the oil-water liquids the correlation of the experiments and calculations is good for both measured parameters - flow velocity and function-indicator of the phase. The stream lines for the turbulent flow presented in Fig. 9 show that a trajectory of a first phase is going nearly parallel to the axis in all mixing layer independent of the density ratio of mixed liquids. In contrast to this, the trajectories of an 
Approaches for Mathematical Modeling and Experimental Study of the Turbulent Flows of Mutually Immiscible Liquids (Oil-Water)

ejected liquid show that intensive mixing of liquids is going by big density ratio: heavy ejected liquid is penetrating the mixing layer in is mixing intensively with a first liquid.

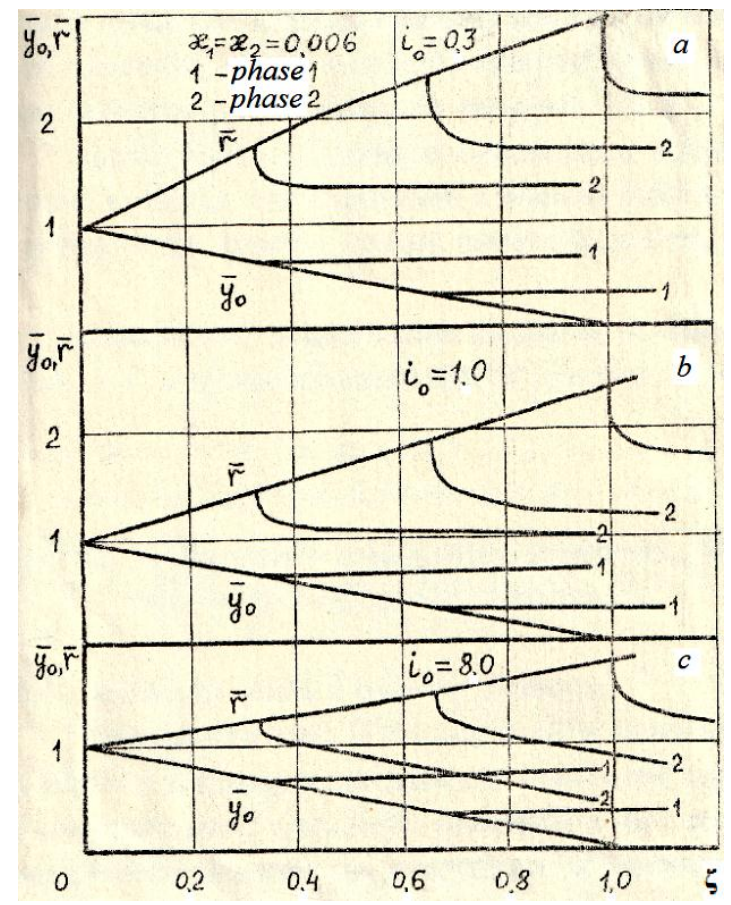

Fig9. The stream lines in a two-phase jet

\section{THE CONCLUSION}

The described mathematical model of the turbulent heterogeneous two-phase jet of two-immiscible liquid was analyzed and supported by numerical simulations and comparison with experimental data. In petroleum business, it may be useful for precise description of the mixing including phase distribution, which is the new valuable opportunity for technical estimations.

\section{ACKNOWLEDGEMENTS}

The author devotes this article to the blessed memory of Prof. Alfred I. Nakorchevski, who was the first his mentor in scientific work. And the author wishes to acknowledge Prof. Torsten H. Fransson for the possibility to work at the Department of Energy Technology, Royal Institute of Technology (KTH), during many years since 2001. Without his support, the author would not have the possibility to work in science due to the bad economic situation and lack of financing of the research in Ukraine. Working experience in the international scientific environment of the KTH was highly valuable for the author in all aspects.

\section{REFERENCES}

[1] NakorchevskiA.I. Heterogeneous turbulent jets. Kyiv: NaukovaDumka, 1980, 142 p.

[2] Ginevskii A.S. The theory of turbulent jets and traces. Integral calculation methods. Moscow: Mashinostroenie, 1969, $400 \mathrm{p}$.

[3] Nakoryakov V.E., Pokusaev B.G., Shreiber I.R. Spreading of the waves in gas- and vapor-liquid media. Novosibirsk: ITF, 1983, 238 p. (In Russian).

[4] Nigmatulin R.I. Fundamentals of mechanics of heterogeneous media. Moscow: Nauka, 1978. - 336 c. (In Russian).

[5] R.I. Nigmatulin and John C. Friedly. Dynamics of Multiphase Media. CRC Press: Volumes $1 \&$ 2. Revised and Augmented Edition (v. 1 \& v. 2), Oct 1, 1990, 878 p.

[6] Nikolay Ivanov Kolev, Multiphase Flow Dynamics 1. 2015. Springer International Publishing. 840 p. (totally 5 volumes by different applications including thermo hydraulics in nuclear energy processes).

[7] Nakorchevski A.I., Kazachkov I.V. Calculation of the heterogeneous turbulent jet. Inbook: Systems of automation of continuous technological processes, Institute of Cybernetics of NASU, 1979,P. 68-79.

[8] Kazachkov I.V. Der turbulente versenkte Strahl von zweier unmischbarenFluessigkeiten. Beitrag. Inst. fuer Kybernetik der Akademie der Wissenschaften der Ukraine. Kiev, 1980. 20p. 
Approaches for Mathematical Modeling and Experimental Study of the Turbulent Flows of Mutually Immiscible Liquids (Oil-Water)

[9] Kazachkov I.V. Mathematical modeling of heterogeneous turbulent jets in cylindrical chamber// Soviet automatic control, 1980, vol.13, jan.-feb., p. 1-6.

[10] KazachkovI.V., NakorchevskiA.I. The stream lines in a turbulent two-phase jet of two immiscible liquids/ Abstracts of the V All SU Meeting on Theor. And Appl. Mechanics. Alma-Ata, 1981 (In Russian).

[11] Kazachkov I.V. Investigation of a turbulent mixing and wall protecting by garnissage in the jet devices working on two immiscible fluids. Candidate of Physics and Mathematics Dissertation (Ph.D.). Kiev T.G. Shevchenko State Univ. 1981. 138p. (in Russian).

[12] NakorchevskiA.I., Basok B.I. Hydrodynamics and heat transfer in heterogeneous systems and devicesof pulsating type. Kyiv: NaukovaDumka, 2001, 348 p.

[13] Kazachkov I.V., PaladinoD. and Sehgal B.R. Ex-vessel coolability of a molten pool by coolant injection from submerged nozzles/ $9^{\text {th }}$ Int. Conf.Nucl. Energy Devel. April 8-12, 2001.Nice, France.

[14] Булат А.Ф. Научно-технические основы создания шахтных когенерационных энергетических комплексов / А.Ф.Булат, И.Ф.Чемерис. - К.: Наук. думка, 2006. - 176 с.

[15] Ландау Л.Д. Гидродинамика / Л.Д. Ландау, Е.М. Лифшиц. - 3-е изд, перераб. - М.: Наука, 1986. $736 \mathrm{c.}$

[16] Ривкин С.Л.Теплофизические свойства воды и водяного пара / С.Л.Ривкин, А.А.Александров. М.:Энергия,1980. - 424 с.

[17] Computational Methods for Multiphase Flow. Andrea Prosperetti. CAMBRIDGE UNIVERSITY PRESS. $2011.742 \mathrm{p}$.

[18] Кириллов.П.Л. Гидродинамические расчёты / П.Л. Кириллов, Ю.С.Юрьев. - М.: ИздАТ, 2009. - 216 c.

[19] Кутателадзе С.С. Экспериментальное исследование пристенных турбулентных течений / С.С. Кутателадзе, Б.П. Миронов, В.Е. Накоряков и др - Новосибирск: Наука, 1975. - 165 с.

[20] Расчет начального участка турбулентной гетерогенной струи. А.И. Накорчевский. Гетерогенные турбулентные струи. К: Наук. Думка, 1980, с. 46-56.

[21] Расчет основного участка турбулентной гетерогенной струи. Там же, с. 61-71.

\section{AUTHORS' BIOGRAPHY}

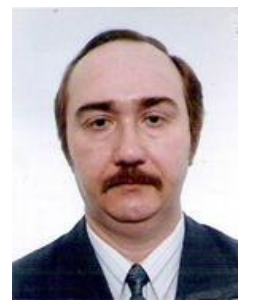

Ivan Vasilievich Kazachkov, was born in 1954 in Olexandriya, Ukraine. He is Mechanical Engineer, Full Doctor of Sciences (1991) in Engineering from the Institute of Physics of the Latvian Academy of Sciences, Ph.D. (Candidate of Physical and Mathematical Sciences, 1981). Full Professor at the Institute of Electrodynamics in Kyiv since 1989. During 1998-2004 worked at the KTH, Energy Technology Dept as Guest Professor, then part-time as Visiting Professor at KTH, teaching numerical methods and doing research in modeling of multiphase systems. Since 2008 till 2014 he was Affiliated Professor at the KTH. Since 2009 - Head of Dept at the Nizhyn Gogol State University, during 2010-2013- also part-time Head of Dept on Heat Power at the Ukrainian Academy of Civil-Communal Economy in Kyiv. The research activities include Parametric Control in Continua, Multiphase Flows, Controlled Film Flow and Granulation of Metals. He has over 200 publications in scientific journals and conferences, 5 patents, 10 monographs and lecture notes, participates in the European research programs and committees. Five PhDs defended their Ph.D. dissertation under his supervision.

Citation: Ivan V. Kazachkov, (2018). Approaches for Mathematical Modeling and Experimental Study of the Turbulent Flows of Mutually Immiscible Liquids (Oil-Water), International Journal of Petroleum and Petrochemical Engineering (IJPPE), 4(1), pp.70-81, DOI: http://dx.doi.org/10.20431/2454-7980.0401008

Copyright: (C) 2018 Ivan V. Kazachkov, (2018). This is an open-access article distributed under the terms of the Creative Commons Attribution License, which permits unrestricted use, distribution, and reproduction in any medium, provided the original author and source are credited 